GLASNIK MATEMATIČKI

Vol. 41(61)(2006), $317-328$

\title{
FRAMES OF SUBMODULES FOR COUNTABLY GENERATED HILBERT $\mathbf{K}(H)$-MODULES
}

\author{
LJILJANA ARAMBAŠIĆ \\ University of Zagreb, Croatia
}

\begin{abstract}
In this paper we introduce frames of submodules for countably generated Hilbert $\mathbf{K}(H)$-modules. We show the correspondence between frames of submodules for a Hilbert $\mathbf{K}(H)$-module $V$ and frames of subspaces for the Hilbert space $V e \subseteq V$, where $e \in \mathbf{K}(H)$ is an arbitrary orthogonal one-dimensional projection. We also establish a relation between their frame operators.
\end{abstract}

\section{INTRODUCTION AND PRELIMINARIES}

Frames of subspaces for a separable Hilbert space have been recently introduced by P. G. Casazza and G. Kutyniok [5]. Frames of subspaces are those families of closed subspaces of a Hilbert space such that taking frames for every subspace (with uniformly bounded frame bounds) and putting them together yields the frame for the whole space. It is shown that frames of subspaces behave as a natural generalization of frames. In this paper we generalize this concept to countably generated Hilbert $\mathbf{K}(H)$-modules and prove that results of Casazza and Kutyniok on frames of subspaces extend to this setting. In particular, Theorem 2.3 shows how to get a frame of submodules for a countably generated Hilbert $\mathbf{K}(H)$-module $V$ from a frame of subspaces for the separable Hilbert space $V e$, and vice versa.

A (right) Hilbert $C^{*}$-module $V$ over a $C^{*}$-algebra $A$ (or a (right) Hilbert $A$-module) is a linear space which is a right $A$-module, together with an $A$ valued inner product $\langle\cdot, \cdot\rangle: V \times V \rightarrow A$ which is $A$-linear in the second and conjugate linear in the first variable such that $V$ is a Banach space with the norm $\|x\|=\|\langle x, x\rangle\|^{1 / 2}$. A Hilbert $A$-module $V$ is countably generated if

2000 Mathematics Subject Classification. 46H25, 46L05, 46L99.

Key words and phrases. $C^{*}$-algebra, Hilbert $C^{*}$-module, compact operator, frame, frame operator, frame of subspaces, frame of submodules. 
there exists a countable set in $V$ such that the set of all its finite $A$-linear combinations is dense in $V$.

A map $T: V \rightarrow W$ between Hilbert $A$-modules $V$ and $W$ for which there exists a map $T^{*}: W \rightarrow V$ such that $\langle T x, y\rangle=\left\langle x, T^{*} y\right\rangle$ for all $x \in V, y \in W$ is called adjointable. An adjointable map is automatically linear, $A$-linear, bounded and its adjoint is unique. We use $\mathbf{B}(V, W)$ to denote the space of all adjointable operators between Hilbert $A$-modules $V$ and $W$, and $\mathbf{B}(V)$ to denote the $C^{*}$-algebra $\mathbf{B}(V, V)$. We use the symbol id $V$ to denote the identity operator on $V$. A closed submodule $W$ of a Hilbert $A$-module $V$ is complementable in $V$ if $W \oplus W^{\perp}=V$, where $W^{\perp}=\{x \in V:\langle x, w\rangle=0, w \in$ $W\}$.

Although Hilbert $C^{*}$-modules behave as a generalization of Hilbert spaces, many of the properties of Hilbert spaces cannot be obtained for general Hilbert $C^{*}$-modules. For example, bounded $A$-linear operators may fail to possess an adjoint. Also, not every closed submodule $W$ of a Hilbert $A$-module $V$ is complementable in $V$. It is known that a closed submodule of a Hilbert $C^{*}$-module $V$ is complementable in $V$ precisely when it is the range of an adjointable operator. For more about Hilbert $C^{*}$-modules see [10] and [14].

Throughout the paper $H$ denotes a separable Hilbert space with an inner product $(\cdot, \cdot)$. By $\mathbf{B}(H)$ resp. $\mathbf{K}(H)$ we denote the $C^{*}$-algebra of all bounded resp. compact linear operators on $H$.

A finite or countable sequence $\left\{f_{i}: i \in I\right\}$ in a Hilbert space $H$ is a frame for $H$ if there are constants $C, D>0$ (the frame bounds) such that

$$
C\|x\|^{2} \leq \sum_{i \in I}\left|\left(x, f_{i}\right)\right|^{2} \leq D\|x\|^{2}, \quad \forall x \in H .
$$

To generalize this definition to the situation of Hilbert $C^{*}$-modules M. Frank and D. Larson rephrased the above inequalities in a suitable way. Therefore, a frame for a countably generated Hilbert $C^{*}$-module $V$ is a finite or countable sequence $\left\{f_{i}: i \in I\right\}$ such that there exist constants $C, D>0$ (the frame bounds) such that

$$
C\langle x, x\rangle \leq \sum_{i \in I}\left\langle x, f_{i}\right\rangle\left\langle f_{i}, x\right\rangle \leq D\langle x, x\rangle, \quad \forall x \in V .
$$

The frame is a Parseval frame if $C=D=1$. A set $\left\{f_{i}: i \in I\right\}$ in a Hilbert $C^{*}$ module $V$ is said to be a Bessel sequence for $V$ if there is a constant $D>0$ (the Bessel bound) such that the inequality $\sum_{i \in I}\left\langle x, f_{i}\right\rangle\left\langle f_{i}, x\right\rangle \leq D\langle x, x\rangle$ holds for every $x \in V$. Frames always exist in countably generated Hilbert $C^{*}$-modules over unital $C^{*}$-algebras and Hilbert $C^{*}$-modules over a $C^{*}$-algebra of (not necessarily all) compact operators. The frame is standard if the sum in the middle of the equality (1.1) converges in norm for every $x \in V$. Since we work only with standard frames in this paper, we will abbreviate the term "standard frames" as "frames". 
The frame transform associated to a frame $\left\{f_{i}: i \in I\right\}$ for a countably generated Hilbert $C^{*}$-module $V$ is a map $\theta: V \rightarrow \ell_{2}(A)$ defined by $\theta(x)=\left(\left\langle f_{i}, x\right\rangle\right)_{i}$. Here $\ell_{2}(A):=\left\{\left(a_{i}\right)_{i}: a_{i} \in A, \sum_{i \in I} a_{i}^{*} a_{i}\right.$ converges in $\left.A\right\}$ is a Hilbert $A$-module with pointwise operations and the inner product defined by $\left\langle\left(a_{i}\right)_{i},\left(b_{i}\right)_{i}\right\rangle=\sum_{i \in I} a_{i}^{*} b_{i}$. The restriction to the sum in the middle of the inequality (1.1) to converge in norm for every $x \in V$ guarantees the existence and the adjointability of the frame transform and an embedding of $V$ onto an orthogonal summand of $\ell_{2}(A)$ ([8, Theorem 4.4]). The operator $S=\theta^{*} \theta \in \mathbf{B}(V)$ is said to be the frame operator for a frame $\left\{f_{i}: i \in I\right\}$. The frame operator is always positive, invertible and it is the unique operator in $\mathbf{B}(V)$ such that the reconstruction formula holds

$$
x=\sum_{i \in I} f_{i}\left\langle S^{-1} f_{i}, x\right\rangle, \quad \forall x \in V .
$$

If $H$ is a separable Hilbert space, then a (finite or countable) family of closed subspaces $\left\{W_{i}: i \in I\right\}$ of $H$ is said to be a frame of subspaces for $H$ with respect to a family of weights $\left\{\lambda_{i}: i \in I\right\}$ (a family of weights $\left\{\lambda_{i}: i \in I\right\}$ is a family of positive real numbers) if there exist constants $C, D>0$ (the frame bounds) such that

$$
C(x, x) \leq \sum_{i \in I} \lambda_{i}^{2}\left(\pi_{i}(x), \pi_{i}(x)\right) \leq D(x, x), \quad \forall x \in H,
$$

where $\pi_{i} \in \mathbf{B}(H), i \in I$ stand for orthogonal projections onto $W_{i}, i \in I$. The frame transform associated to a frame of subspaces $\left\{W_{i}: i \in I\right\}$ for $H$ with respect to a family of weights $\left\{\lambda_{i}: i \in I\right\}$ is defined by

$$
\theta_{W, \lambda} \in \mathbf{B}\left(H, \bigoplus_{i \in I} W_{i}\right), \quad \theta_{W, \lambda}(x)=\left(\lambda_{i} \pi_{i}(x)\right)_{i},
$$

where $\bigoplus_{i \in I} W_{i}=\left\{\left(w_{i}\right)_{i}: w_{i} \in W_{i}, \sum_{i \in I}\left\|w_{i}\right\|^{2}<\infty\right\}$. The operator $S_{W, \lambda}=$ $\theta_{W, \lambda}^{*} \theta_{W, \lambda} \in \mathbf{B}(H)$ is called the frame operator for $\left\{W_{i}: i \in I\right\}$ and $\left\{\lambda_{i}: i \in I\right\}$.

Now we state some basic properties about Hilbert $\mathbf{K}(H)$-modules. If $V$ is a Hilbert $\mathbf{K}(H)$-module, then each bounded $\mathbf{K}(H)$-linear operator on $V$ is necessarily adjointable, and each closed submodule $W \subseteq V$ is complementable in $V$.

An element $v$ of a Hilbert $\mathbf{K}(H)$-module $V$ is a basic vector if $e=\langle v, v\rangle \in$ $\mathbf{K}(H)$ is an orthogonal one-dimensional projection. A system of basic vectors $\left\{v_{i}: i \in I\right\}$ in $V$ is an orthonormal basis for $V$ if $\left\langle v_{i}, v_{j}\right\rangle=0$ for all $i \neq j$, and if it generates a dense submodule of $V$. Every Hilbert $\mathbf{K}(H)$-module possesses an orthonormal basis. (Clearly, an arbitrary Hilbert $C^{*}$-module need not possess an orthonormal basis; actually, if $V$ possesses an orthonormal basis then the $C^{*}$-algebra generated by $\{\langle x, y\rangle: x, y \in V\}$ is a $C C R$-algebra [1].) Every orthonormal basis for a Hilbert $\mathbf{K}(H)$-module $V$ is a Parseval frame for $V$. 
Furthermore, if $e \in \mathbf{K}(H)$ is an orthogonal one-dimensional projection, then $V e:=\{x e: x \in V\}$ is a Hilbert space with respect to the inner product $(x, y)=\operatorname{tr}(\langle y, x\rangle)$ where 'tr' means the trace. It is easy to see that $\langle x, y\rangle=$ $(y, x) e$ for all $x, y \in V e . V e$ is an invariant subspace for each $T$ in $\mathbf{B}(V)$ and the map

$$
T: \mathbf{B}(V) \rightarrow \mathbf{B}(V e), \quad T \mapsto T \mid V e
$$

establishes an isomorphism between $C^{*}$-algebras $\mathbf{B}(V)$ and $\mathbf{B}(V e)$. For more details about Hilbert $\mathbf{K}(H)$-modules we refer to $[3,11,13]$.

\section{Definition AND BASIC PROPERTIES}

In this section we define and discuss frames of submodules for countably generated Hilbert $\mathbf{K}(H)$-modules. We show that, for a fixed orthogonal one-dimensional projection $e \in \mathbf{K}(H)$, a family $\left\{W_{i}: i \in I\right\}$ is a frame of submodules for a Hilbert $\mathbf{K}(H)$-module $V$ if and only if $\left\{W_{i} e: i \in I\right\}$ is a frame of subspaces for the Hilbert space $V e$.

Definition 2.1. Let $V$ be a countably generated Hilbert $\mathbf{K}(H)$-module and $I \subseteq \mathbb{N}$ finite or countable. Let $\left\{\lambda_{i}: i \in I\right\}$ be a family of weights. $A$ family of closed submodules $\left\{W_{i}: i \in I\right\}$ of $V$ is a frame of submodules for $V$ with respect to $\left\{\lambda_{i}: i \in I\right\}$, if there exist constants $C, D>0$ such that

$$
C\langle x, x\rangle \leq \sum_{i \in I} \lambda_{i}^{2}\left\langle\pi_{i}(x), \pi_{i}(x)\right\rangle \leq D\langle x, x\rangle, \quad \forall x \in V,
$$

where $\pi_{i} \in \mathbf{B}(V), i \in I$ denote orthogonal projections on $W_{i}$, and the convergence of the sum in the middle of (2.1) is in norm. We call the constants $C$ and $D$ the frame bounds for the frame of submodules. If $C=D=1$ we say that a frame of submodules is a Parseval frame of submodules.

We say that a family of submodules $\left\{W_{i}: i \in I\right\}$ is a Bessel sequence of submodules for $V$ with respect to $\left\{\lambda_{i}: i \in I\right\}$ if there exists a constant $D>0$ (the Bessel bound) such that the right-hand side of (2.1) holds.

Let us notice that, since $V$ is a Hilbert $\mathbf{K}(H)$-module, every closed submodule of $V$ has an orthogonal complement, so projections $\pi_{i}$ are well defined and $\pi_{i} \in \mathbf{B}(V)$ for all $i \in I$. Also, observe that if $\left\{W_{i}: i \in I\right\}$ is a Bessel sequence for $V$ with respect to a family of weights $\left\{\lambda_{i}: i \in I\right\}$, then a family $\{V\} \cup\left\{W_{i}: i \in I\right\}$ is a frame of submodules for $V$ with respect to a family of weights $\left\{\lambda_{0}\right\} \cup\left\{\lambda_{i}: i \in I\right\}$, where $\lambda_{0}$ is an arbitrary positive number. If $D$ is a Bessel bound, then the frame bounds will be $\lambda_{0}^{2}$ and $\lambda_{0}^{2}+D$.

In Hilbert spaces the condition (2.1) is necessary and sufficient so that, putting together frames for every component of the frame of subspaces, we get a frame for the whole space ([5, Theorem 3.2]). The same result holds in Hilbert $\mathbf{K}(H)$-modules. 
THEOREM 2.2. Let $V$ be a countably generated Hilbert $\mathbf{K}(H)$-module, $\left\{W_{i}: i \in I\right\}$ a family of closed submodules of $V$ and $\left\{\lambda_{i}: i \in I\right\}$ a family of weights. For every $i \in I$ let $\left\{f_{i j}: j \in J_{i}\right\}$ be a (Parseval) frame for $W_{i}$ with frame bounds $A_{i}$ and $B_{i}$. Let $\left\{e_{i j}: j \in K_{i}\right\}$ be an orthonormal basis for $W_{i}$. Suppose that

$$
0<A:=\inf _{i \in I} A_{i} \leq B:=\sup _{i \in I} B_{i}<\infty .
$$

The following statements are mutually equivalent:

(1) $\left\{\lambda_{i} f_{i j}: i \in I, j \in J_{i}\right\}$ is a (Parseval) frame for $V$.

(2) $\left\{\lambda_{i} e_{i j}: i \in I, j \in K_{i}\right\}$ is a (Parseval) frame for $V$.

(3) $\left\{W_{i}: i \in I\right\}$ is a (Parseval) frame of submodules for $V$ with respect to $\left\{\lambda_{i}: i \in I\right\}$.

Proof. For every $i \in I$ and $x \in W_{i}$ we have

$$
A_{i}\langle x, x\rangle \leq \sum_{j \in J_{i}}\left\langle x, f_{i j}\right\rangle\left\langle f_{i j}, x\right\rangle \leq B_{i}\langle x, x\rangle .
$$

Since $\pi_{i}\left(f_{i j}\right)=f_{i j}$ and then $\left\langle\lambda_{i} \pi_{i}(x), f_{i j}\right\rangle=\left\langle x, \lambda_{i} f_{i j}\right\rangle$ for all $i \in I, j \in J_{i}$, we have

$$
\begin{gathered}
A \sum_{i \in I} \lambda_{i}^{2}\left\langle\pi_{i}(x), \pi_{i}(x)\right\rangle \leq \sum_{i \in I} A_{i}\left\langle\lambda_{i} \pi_{i}(x), \lambda_{i} \pi_{i}(x)\right\rangle \\
\leq \sum_{i \in I} \sum_{j \in J_{i}}\left\langle\lambda_{i} \pi_{i}(x), f_{i j}\right\rangle\left\langle f_{i j}, \lambda_{i} \pi_{i}(x)\right\rangle=\sum_{i \in I} \sum_{j \in J_{i}}\left\langle x, \lambda_{i} f_{i j}\right\rangle\left\langle\lambda_{i} f_{i j}, x\right\rangle \\
\leq \sum_{i \in I} B_{i}\left\langle\lambda_{i} \pi_{i}(x), \lambda_{i} \pi_{i}(x)\right\rangle \leq B \sum_{i \in I} \lambda_{i}^{2}\left\langle\pi_{i}(x), \pi_{i}(x)\right\rangle .
\end{gathered}
$$

$(1) \Longrightarrow(3):$ If $\left\{\lambda_{i} f_{i j}: i \in I, j \in J_{i}\right\}$ is a frame for $V$ with frame bounds $C$ and $D$, then for all $x \in V$ we have

$$
\begin{aligned}
& \sum_{i \in I} \lambda_{i}^{2}\left\langle\pi_{i}(x), \pi_{i}(x)\right\rangle \leq \frac{1}{A} \sum_{i \in I} \sum_{j \in J_{i}}\left\langle x, \lambda_{i} f_{i j}\right\rangle\left\langle\lambda_{i} f_{i j}, x\right\rangle \leq \frac{D}{A}\langle x, x\rangle, \\
& \sum_{i \in I} \lambda_{i}^{2}\left\langle\pi_{i}(x), \pi_{i}(x)\right\rangle \geq \frac{1}{B} \sum_{i \in I} \sum_{j \in J_{i}}\left\langle x, \lambda_{i} f_{i j}\right\rangle\left\langle\lambda_{i} f_{i j}, x\right\rangle \geq \frac{C}{B}\langle x, x\rangle .
\end{aligned}
$$

Therefore, $\left\{W_{i}: i \in I\right\}$ is a frame of submodules for $V$ with respect to $\left\{\lambda_{i}: i \in I\right\}$ with frame bounds $\frac{C}{B}$ and $\frac{D}{A}$.

(3) $\Longrightarrow(1):$ If $\left\{W_{i}: i \in I\right\}$ is a frame of submodules for $V$ with frame bounds $C$ and $D$, then

$$
\begin{gathered}
A C\langle x, x\rangle \leq A \sum_{i \in I} \lambda_{i}^{2}\left\langle\pi_{i}(x), \pi_{i}(x)\right\rangle \leq \sum_{i \in I} \sum_{j \in J_{i}}\left\langle x, \lambda_{i} f_{i j}\right\rangle\left\langle\lambda_{i} f_{i j}, x\right\rangle \\
\leq B \sum_{i \in I} \lambda_{i}^{2}\left\langle\pi_{i}(x), \pi_{i}(x)\right\rangle \leq B D\langle x, x\rangle, \quad \forall x \in V .
\end{gathered}
$$


Hence, $\left\{\lambda_{i} f_{i j}: i \in I, j \in J_{i}\right\}$ is a frame for $V$ with frame bounds $A C$ and $B D$.

$(2) \Leftrightarrow(3)$ is a special case of $(1) \Leftrightarrow(3)$ since orthonormal bases are Parseval frames.

Notice that, if $A=B=1$ in (2.2), then it follows from the above proof that the frame bounds of $\left\{\lambda_{i} f_{i j}: i \in I, j \in J_{i}\right\},\left\{\lambda_{i} e_{i j}: i \in I, j \in K_{i}\right\}$ and $\left\{W_{i}: i \in I\right\}$ are the same. In particular, this happens when we choose Parseval frames for every $W_{i}$, so in this case it holds: $\left\{\lambda_{i} f_{i j}: i \in I, j \in J_{i}\right\}$ is a Parseval frame for $V$ if and only if $\left\{\lambda_{i} e_{i j}: i \in I, j \in K_{i}\right\}$ is a Parseval frame for $V$ if and only if $\left\{W_{i}: i \in I\right\}$ is a Parseval frame of submodules for $V$.

TheOrem 2.3. Let $V$ be a countably generated Hilbert $\mathbf{K}(H)$-module, $\left\{W_{i}: i \in I\right\}$ a family of closed submodules of $V$ and $\left\{\lambda_{i}: i \in I\right\}$ a family of weights. Let $e \in \mathbf{K}(H)$ be an orthogonal one-dimensional projection. The following statements are mutually equivalent:

(1) $\left\{W_{i}: i \in I\right\}$ is a frame of submodules for a Hilbert $\mathbf{K}(H)$-module $V$ with respect to $\left\{\lambda_{i}: i \in I\right\}$ with frame bounds $C$ and $D$.

(2) $\left\{W_{i} e: i \in I\right\}$ is a frame of subspaces for the Hilbert space Ve with respect to $\left\{\lambda_{i}: i \in I\right\}$ with frame bounds $C$ and $D$.

Proof. For every $i \in I$ let $\left\{e_{i j}: j \in J_{i}\right\} \subseteq V e$ be an ortonormal basis for $W_{i}$. By [3, Remark 4(e)], $\left\{e_{i j}: j \in J_{i}\right\}$ is also an orthonormal basis for the Hilbert space $W_{i} e$. The frame bounds are $A_{i}=B_{i}=1$ for all $i \in I$, so $(2.2)$ is fulfilled with $A=B=1$. It follows from Theorem 2.2 that $\left\{W_{i}: i \in I\right\}$ is a frame of submodules for $V$ with respect to $\left\{\lambda_{i}: i \in I\right\}$ if and only if $\left\{\lambda_{i} e_{i j}: i \in I, j \in J_{i}\right\}$ is a frame for $V$ with the same frame bounds. Then, by [1, Theorem 3.4], $\left\{\lambda_{i} e_{i j}: i \in I, j \in J_{i}\right\}$ is a frame for a Hilbert module $V$ if and only if $\left\{\lambda_{i} e_{i j}: i \in I, j \in J_{i}\right\}$ is a frame for the Hilbert space $V e$ with the same frame bounds. Finally, by [5, Theorem 3.2], $\left\{\lambda_{i} e_{i j}: i \in I, j \in J_{i}\right\}$ is a frame for the Hilbert space $V e$ if and only if $\left\{W_{i} e: i \in I\right\}$ is a frame of subspaces for the Hilbert space $V e$ with respect to $\left\{\lambda_{i}: i \in I\right\}$. Again, since $A=B=1$, the frame bounds are preserved.

Since frames of subspaces always exist in separable Hilbert spaces, it follows from this theorem that frames of submodules exist in countably generated Hilbert $\mathbf{K}(H)$-modules. Indeed, let $V$ be a countably generated Hilbert $\mathbf{K}(H)$-module and $e \in \mathbf{K}(H)$ an orthogonal one-dimensional projection. Let $\left\{W_{i}^{e}: i \in I\right\}$ be a frame of subspaces for the Hilbert space $V e$ with respect to a family of weights $\left\{\lambda_{i}: i \in I\right\}$. For every $i \in I$ we define $W_{i}$ to be a closed submodule of $V$ generated by $W_{i}^{e}$. Then $W_{i} e=W_{i}^{e}$, so by Theorem 2.3 $\left\{W_{i}: i \in I\right\}$ is a frame of submodules for $V$ with respect to $\left\{\lambda_{i}: i \in I\right\}$. Also, we see in the next proposition that unitary operators on $V$ preserve (Parseval) frames of submodules. 
Proposition 2.4. Let $V$ be a countably generated Hilbert $\mathbf{K}(H)$-module and $\left\{W_{i}: i \in I\right\}$ a frame of submodules for $V$ with respect to a family of weights $\left\{\lambda_{i}: i \in I\right\}$ with frame bounds $C$ and $D$. For every unitary operator $T \in \mathbf{B}(V)$ the family $\left\{T W_{i}: i \in I\right\}$ is a frame of submodules for $V$ with respect to the family of weights $\left\{\lambda_{i}: i \in I\right\}$ with frame bounds $C$ and $D$.

Proof. Let $\pi_{W_{i}}$ and $\pi_{T W_{i}}$ denote the orthogonal projections on $W_{i}$ and $T W_{i}$, respectively. $T$ is unitary, hence $\pi_{T W_{i}}=T \pi_{W_{i}} T^{*}$ and $\langle T x, T x\rangle=$ $\langle x, x\rangle=\left\langle T^{*} x, T^{*} x\right\rangle, x \in V$. Then for every $x \in V$ we have

$$
\begin{aligned}
& \sum_{i \in I} \lambda_{i}^{2}\left\langle\pi_{T W_{i}}(x), \pi_{T W_{i}}(x)\right\rangle=\sum_{i \in I} \lambda_{i}^{2}\left\langle T \pi_{W_{i}} T^{*}(x), T \pi_{W_{i}} T^{*}(x)\right\rangle \\
& =\sum_{i \in I} \lambda_{i}^{2}\left\langle\pi_{W_{i}} T^{*}(x), \pi_{W_{i}} T^{*}(x)\right\rangle \leq D\left\langle T^{*}(x), T^{*}(x)\right\rangle=D\langle x, x\rangle,
\end{aligned}
$$

and similarly $\sum_{i \in I} \lambda_{i}^{2}\left\langle\pi_{T W_{i}}(x), \pi_{T W_{i}}(x)\right\rangle \geq C\langle x, x\rangle$.

\section{The Frame transform AND THE FRAme OPERATOR}

In this section we associate the frame transform and the frame operator to a frame of submodules. We show that the frame operator $S_{W, \lambda}$ for a frame of submodules $\left\{W_{i}: i \in I\right\}$ for a Hilbert $\mathbf{K}(H)$-module $V$ is the unique extension in $\mathbf{B}(V)$ of the frame operator $S_{W e, \lambda} \in \mathbf{B}(V e)$ for a frame of subspaces $\left\{W_{i} e: i \in I\right\}$ for the Hilbert space $V e$.

If $\left\{W_{i}: i \in I\right\}$ is a family of Hilbert $A$-modules, then we denote

$$
\bigoplus_{i \in I} W_{i}:=\left\{\left(w_{i}\right)_{i}: w_{i} \in W_{i}, \sum_{i \in I}\left\langle w_{i}, w_{i}\right\rangle \text { converges in } A\right\} .
$$

$\bigoplus_{i \in I} W_{i}$ is a Hilbert $A$-module with pointwise operations and the inner product $\left\langle\left(w_{i}\right)_{i},\left(z_{i}\right)_{i}\right\rangle=\sum_{i \in I}\left\langle w_{i}, z_{i}\right\rangle$.

We first prove a preparatory lemma.

Lemma 3.1. Let $\left\{W_{i}: i \in I\right\}$ be a Bessel sequence of submodules for a countably generated Hilbert $\mathbf{K}(H)$-module $V$ with respect to a family of weights $\left\{\lambda_{i}: i \in I\right\}$. Then the following statements hold:

(1) The series $\sum_{i \in I} \lambda_{i} w_{i}$ converges for every $\left(w_{i}\right)_{i \in I} \in \bigoplus_{i \in I} W_{i}$.

(2) For every $x \in V$ we have $\left(\lambda_{i} \pi_{i}(x)\right)_{i \in I} \in \bigoplus_{i \in I} W_{i}$.

Proof. (1) Let $D$ be a Bessel bound. Let $J$ be an arbitrarily chosen finite subset of $I$ and $g_{J}=\sum_{i \in J} \lambda_{i} w_{i}$. Then

$$
\begin{aligned}
\left\|g_{J}\right\|^{4} & =\left\|\left\langle g_{J}, g_{J}\right\rangle\right\|^{2}=\left\|\left\langle g_{J}, \sum_{i \in J} \lambda_{i} w_{i}\right\rangle\right\|^{2}=\left\|\sum_{i \in J} \lambda_{i}\left\langle g_{J}, w_{i}\right\rangle\right\|^{2} \\
& =\left\|\sum_{i \in J} \lambda_{i}\left\langle g_{J}, \pi_{i}\left(w_{i}\right)\right\rangle\right\|^{2}=\left\|\sum_{i \in J}\left\langle\lambda_{i} \pi_{i}\left(g_{J}\right), w_{i}\right\rangle\right\|^{2}
\end{aligned}
$$




$$
\begin{gathered}
\leq\left\|\sum_{i \in J}\left\langle\lambda_{i} \pi_{i}\left(g_{J}\right), \lambda_{i} \pi_{i}\left(g_{J}\right)\right\rangle\right\| \cdot\left\|\sum_{i \in J}\left\langle w_{i}, w_{i}\right\rangle\right\| \\
\leq D\left\|g_{J}\right\|^{2} \cdot\left\|\sum_{i \in J}\left\langle w_{i}, w_{i}\right\rangle\right\|,
\end{gathered}
$$

wherefrom

$$
\left\|g_{J}\right\|^{2}=\left\|\sum_{i \in J} \lambda_{i} w_{i}\right\|^{2} \leq D\left\|\sum_{i \in J}\left\langle w_{i}, w_{i}\right\rangle\right\|^{2} .
$$

Since $\left(w_{i}\right)_{i} \in \bigoplus_{i \in I} W_{i}$, the series $\sum_{i \in J}\left\langle w_{i}, w_{i}\right\rangle$ converges in norm; therefore $\sum_{i \in I} \lambda_{i} w_{i}$ also converges. ules.

(2) It follows directly from the definition of a Bessel sequence of submod-

If $\left\{W_{i}: i \in I\right\}$ is a Bessel sequence of submodules with a Bessel bound $D$, then the preceding lemma ensures the well-definedness of the operator

$$
\theta_{W, \lambda}: V \rightarrow \bigoplus_{i \in I} W_{i}, \quad \theta_{W, \lambda}(x)=\left(\lambda_{i} \pi_{i}(x)\right)_{i \in I} .
$$

Since $\left\langle\theta_{W, \lambda}(x), \theta_{W, \lambda}(x)\right\rangle \leq D\langle x, x\rangle$ for all $x \in V, \theta_{W, \lambda}$ is bounded and $\left\|\theta_{W, \lambda}\right\| \leq \sqrt{D}$. Further, for every $\left(w_{i}\right)_{i \in I} \in \bigoplus_{i \in I} W_{i}$ we have

$$
\left\langle\theta_{W, \lambda}(x),\left(w_{i}\right)_{i}\right\rangle=\sum_{i \in I} \lambda_{i}\left\langle\pi_{i}(x), w_{i}\right\rangle=\sum_{i \in I} \lambda_{i}\left\langle x, w_{i}\right\rangle=\left\langle x, \sum_{i \in I} \lambda_{i} w_{i}\right\rangle,
$$

hence $\theta_{W, \lambda} \in \mathbf{B}\left(V, \bigoplus_{i \in I} W_{i}\right)$ and $\theta_{W, \lambda}^{*}\left(\left(w_{i}\right)_{i}\right)=\sum_{i \in I} \lambda_{i} w_{i}$.

In the following theorem we prove that $\theta_{W, \lambda}$ characterizes a frame of submodules for a Hilbert $\mathbf{K}(H)$-module just as in the case of a Hilbert space (see [5, Theorem 3.12]).

TheOREm 3.2. Let $V$ be a countably generated Hilbert $\mathbf{K}(H)$-module, $\left\{W_{i}: i \in I\right\}$ a family of closed submodules of $V$ and $\left\{\lambda_{i}: i \in I\right\}$ a family of weights. The following statements are mutually equivalent:

(1) $\left\{W_{i}: i \in I\right\}$ is a frame of submodules for $V$ with respect to $\left\{\lambda_{i}: i \in I\right\}$.

(2) $\theta_{W, \lambda} \in \mathbf{B}\left(V, \bigoplus_{i \in I} W_{i}\right)$ and $\theta_{W, \lambda}^{*}$ is surjective.

(3) $\theta_{W, \lambda} \in \mathbf{B}\left(V, \bigoplus_{i \in I} W_{i}\right)$ and $\theta_{W, \lambda}$ is bounded below, i.e. there is $m>0$ such that $\left\|\theta_{W, \lambda}(x)\right\| \geq m\|x\|$ for all $x \in V$.

In particular, $\left\{W_{i}: i \in I\right\}$ is a Bessel sequence of submodules for $V$ with respect to $\left\{\lambda_{i}: i \in I\right\}$ if and only if $\theta_{W, \lambda} \in \mathbf{B}\left(V, \bigoplus_{i \in I} W_{i}\right)$.

Proof. It is obvious that $\left\{W_{i}: i \in I\right\}$ is a frame of submodules for $V$ with respect to $\left\{\lambda_{i}: i \in I\right\}$ if and only if $\theta_{W, \lambda} \in \mathbf{B}\left(V, \bigoplus_{i \in I} W_{i}\right)$ and $\theta_{W, \lambda}$ is bounded below with respect to the inner product, since

$$
\left\langle\theta_{W, \lambda}(x), \theta_{W, \lambda}(x)\right\rangle=\left\langle\left(\lambda_{i} \pi_{i}(x)\right)_{i},\left(\lambda_{i} \pi_{i}(x)\right)_{i}\right\rangle=\sum_{i \in I} \lambda_{i}^{2}\left\langle\pi_{i}(x), \pi_{i}(x)\right\rangle
$$


for all $x \in V$. If $\theta_{W, \lambda} \in \mathbf{B}\left(V, \bigoplus_{i \in I} W_{i}\right)$, then $\theta_{W, \lambda}$ is bounded below with respect to the inner product if and only if $\theta_{W, \lambda}$ is bounded below with respect to the norm if and only if $\theta_{W, \lambda}^{*}$ is surjective (see [1, Proposition 2.1]). This completes the proof.

Definition 3.3. Let $V$ be a countably generated Hilbert $\mathbf{K}(H)$-module and $\left\{W_{i}: i \in I\right\}$ a frame of submodules for $V$ with respect to a family of weights $\left\{\lambda_{i}: i \in I\right\}$. The frame transform for $\left\{W_{i}: i \in I\right\}$ and $\left\{\lambda_{i}: i \in I\right\}$ is the operator

$$
\theta_{W, \lambda} \in \mathbf{B}\left(V, \bigoplus_{i \in I} W_{i}\right), \quad \theta_{W, \lambda}(x)=\left(\lambda_{i} \pi_{i}(x)\right)_{i \in I} .
$$

The frame operator for $\left\{W_{i}: i \in I\right\}$ and $\left\{\lambda_{i}: i \in I\right\}$ is the operator

$$
S_{W, \lambda}=\theta_{W, \lambda}^{*} \theta_{W, \lambda} \in \mathbf{B}(V), \quad S_{W, \lambda}(x)=\sum_{i \in I} \lambda_{i}^{2} \pi_{i}(x) .
$$

Proposition 3.4. Let $V$ be a countably generated Hilbert $\mathbf{K}(H)$-module and $\left\{W_{i}: i \in I\right\}$ a frame of submodules for $V$ with respect to a family of weights $\left\{\lambda_{i}: i \in I\right\}$ with frame bounds $C$ and $D$. The frame operator $S_{W, \lambda} \in$ $\mathbf{B}(V)$ is a positive invertible operator such that $C \cdot \operatorname{id}_{V} \leq S_{W, \lambda} \leq D \cdot \operatorname{id}_{V}$. In particular, $\left\{W_{i}: i \in I\right\}$ is a Parseval frame of submodules for $V$ with respect to $\left\{\lambda_{i}: i \in I\right\}$ if and only if $S_{W, \lambda}=\mathrm{id}_{V}$. Further, we have the reconstruction formula

$$
x=\sum_{i \in I} \lambda_{i}^{2} S_{W, \lambda}^{-1} \pi_{i}(x), \quad \forall x \in V .
$$

Proof. Since $\left\{W_{i}: i \in I\right\}$ is a frame of submodules and

$$
\left\langle S_{W, \lambda}(x), x\right\rangle=\left\langle\sum_{i \in I} \lambda_{i}^{2} \pi_{i}(x), x\right\rangle=\sum_{i \in I} \lambda_{i}^{2}\left\langle\pi_{i}(x), \pi_{i}(x)\right\rangle, \quad \forall x \in V,
$$

we have $C\langle x, x\rangle \leq\left\langle S_{W, \lambda}(x), x\right\rangle \leq D\langle x, x\rangle$ for all $x \in V$. By [10, Lemma 4.1], it follows that $C \cdot \operatorname{id}_{V} \leq S_{W, \lambda} \leq D \cdot \operatorname{id}_{V}$. In particular, $S_{W, \lambda}$ is positive and bounded below, therefore bijective ([1, Proposition 2.1]). Now the reconstruction formula follows:

$$
x=S_{W, \lambda}^{-1} S_{W, \lambda}(x)=S_{W, \lambda}^{-1}\left(\sum_{i \in I} \lambda_{i}^{2} \pi_{i}(x)\right)=\sum_{i \in I} \lambda_{i}^{2} S_{W, \lambda}^{-1} \pi_{i}(x), \quad \forall x \in V .
$$

Proposition 3.5. Let $V$ be a countably generated Hilbert $\mathbf{K}(H)$-module and $\left\{W_{i}: i \in I\right\}$ a frame of submodules for $V$ with respect to a family of weights $\left\{\lambda_{i}: i \in I\right\}$. For every $i \in I$ let $\left\{f_{i j}: j \in J_{i}\right\}$ be a Parseval frame for 
$W_{i}$. Let $S_{W, \lambda}$ be the frame operator for $\left\{W_{i}: i \in I\right\}$ and $\left\{\lambda_{i}: i \in I\right\}$, and $S_{\lambda f}$ the frame operator for the frame $\left\{\lambda_{i} f_{i j}: i \in I, j \in J_{i}\right\}$. Then $S_{W, \lambda}=S_{\lambda f}$ and

$$
\sum_{i \in I} \lambda_{i}^{2} S_{W, \lambda}^{-1} \pi_{i}(x)=\sum_{i \in I} \sum_{j \in J_{i}} \lambda_{i} S_{\lambda f}^{-1}\left(f_{i j}\right)\left\langle\lambda_{i} f_{i j}, x\right\rangle, \quad \forall x \in V .
$$

Proof. We first notice that by Theorem $2.2\left\{\lambda_{i} f_{i j}: i \in I, j \in J_{i}\right\}$ is indeed a frame for $V$ and $S_{\lambda f}(x)=\sum_{i \in I} \sum_{j \in J_{i}} \lambda_{i} f_{i j}\left\langle\lambda_{i} f_{i j}, x\right\rangle$ for all $x \in V$.

Since $\left\{f_{i j}: j \in J_{i}\right\}$ is a Parseval frame for $W_{i}$ and since $\pi_{i}(x) \in W_{i}$ for all $x \in V$, we have

$$
\left\langle\pi_{i}(x), \pi_{i}(x)\right\rangle=\sum_{j \in J_{i}}\left\langle\pi_{i}(x), f_{i j}\right\rangle\left\langle f_{i j}, \pi_{i}(x)\right\rangle=\sum_{j \in J_{i}}\left\langle x, f_{i j}\right\rangle\left\langle f_{i j}, x\right\rangle .
$$

It follows from here that

$$
\begin{aligned}
\left\langle S_{W, \lambda}(x), x\right\rangle & =\sum_{i \in I} \lambda_{i}^{2}\left\langle\pi_{i}(x), \pi_{i}(x)\right\rangle=\sum_{i \in I} \sum_{j \in J_{i}} \lambda_{i}^{2}\left\langle x, f_{i j}\right\rangle\left\langle f_{i j}, x\right\rangle \\
& =\sum_{i \in I} \sum_{j \in J_{i}}\left\langle x, \lambda_{i} f_{i j}\right\rangle\left\langle\lambda_{i} f_{i j}, x\right\rangle=\left\langle S_{\lambda f}(x), x\right\rangle,
\end{aligned}
$$

for all $x \in V$. Since the operator $S_{W, \lambda}-S_{\lambda f}$ is self-adjoint, it follows from $\left\langle\left(S_{W, \lambda}-S_{\lambda f}\right) x, x\right\rangle=0, x \in V$ that $S_{W, \lambda}=S_{\lambda f}$. By Proposition 3.4, $x=$ $\sum_{i \in I} \lambda_{i}^{2} S_{W, \lambda}^{-1} \pi_{i}(x)$. On the other hand, for every $x \in V$ we have

$$
x=S_{\lambda f}^{-1} S_{\lambda f}(x)=S_{\lambda f}^{-1}\left(\sum_{i \in I} \sum_{j \in J_{i}} \lambda_{i} f_{i j}\left\langle\lambda_{i} f_{i j}, x\right\rangle\right)=\sum_{i \in I} \sum_{j \in J_{i}} \lambda_{i} S_{\lambda f}^{-1} f_{i j}\left\langle\lambda_{i} f_{i j}, x\right\rangle,
$$

and (3.2) is proved.

The frame operators for the associated frames of submodules and subspaces (in the sense of Theorem 2.3) are related in an expected way.

TheOREM 3.6. Let $V$ be a countably generated Hilbert $\mathbf{K}(H)$-module, $\left\{W_{i}: i \in I\right\}$ a frame of submodules for $V$ with respect to a family of weights $\left\{\lambda_{i}: i \in I\right\}$. Let $e \in \mathbf{K}(H)$ be an orthogonal one-dimensional projection. Let $S_{W, \lambda} \in \mathbf{B}(V)$ and $S_{W e, \lambda} \in \mathbf{B}(V e)$ be the frame operators associated to the frame of submodules $\left\{W_{i}: i \in I\right\}$ and the frame of subspaces $\left\{W_{i} e: i \in I\right\}$, respectively. Then we have $S_{W, \lambda} \mid V e=S_{W e, \lambda}$ and $S_{W, \lambda}$ is the unique extension in $\mathbf{B}(V)$ of the operator $S_{W e, \lambda}$.

Proof. For every $i \in I$ let $\pi_{W_{i}} \in \mathbf{B}(V)$ and $\pi_{W_{i} e} \in \mathbf{B}(V e)$ be the orthogonal projections on $W_{i}$ and $W_{i} e$, respectively. Since $\pi_{W_{i}} \mid\left(W_{i} e\right)=\pi_{W_{i}} e$, we have

$$
S_{W, \lambda}(x e)=\sum_{i \in I} \lambda_{i}^{2} \pi_{W_{i}}(x e)=\sum_{i \in I} \lambda_{i}^{2} \pi_{W_{i} e}(x e)=S_{W e, \lambda}(x e)
$$

for all $x e \in V e$. The uniqueness follows from the fact that the map (1.4) is an isomorphism. 
REMARK 3.7. The concept of frames of submodules can be introduced for general countably generated Hilbert $C^{*}$-modules $V$ in the following way: a frame of submodules for $V$ is a family $\left\{W_{i}: i \in I\right\}$ of complementable submodules of $V$ for which there exist $C, D>0$ such that (2.1) holds for every $x \in V$. (The condition of complementability ensures that $\pi_{i} \in \mathbf{B}(V), i \in I$.) Some of the proofs given in this paper also work in this more general situation, and some other statements from [5] can be directly checked, by following proofs for Hilbert spaces. Obviously, our main results which establish relations between frames of submodules for $V$ and frames of subspaces for $V e$ do not hold (Theorems 2.3 and 3.6). Since these results were essential to obtain the existence (and examples) of frames of submodules for countably generated Hilbert $\mathbf{K}(H)$-modules, we do not know if the frames of submodules exist in general countably generated Hilbert $C^{*}$-modules. Several examples of frames of subspaces for separable Hilbert spaces are constructed in [5]. Unfortunately, we cannot apply these constructions to a Hilbert $C^{*}$-module situation since we do not know if the submodules obtained in these ways will be complementable (unless we work with Hilbert $C^{*}$-modules over compact operators).

\section{ACKNOWLEDGEMENT.}

I would like to thank Professors Damir Bakić and Boris Guljaš for valuable suggestions and comments.

\section{REFERENCES}

[1] Lj. Arambašić, On frames for countably generated Hilbert $C^{*}$-modules, Proc. Amer. Math. Soc. 135 (2007), 469-478.

[2] M. S. Asgari and A. Khosravi, Frames and bases of subspaces in Hilbert spaces, J. Math. Anal. Appl. 308 (2005), 541-553.

[3] D. Bakić and B. Guljaš, Hilbert $C^{*}$-modules over $C^{*}$-algebras of compact operators, Acta Sci. Math. (Szeged) 68 (2002), 249-269.

[4] P. G. Casazza, The art of frame theory, Taiwanese J. Math 4 (2000), 129-201.

[5] P. G. Casazza and G. Kutyniok, Frames of subspaces, Wavelets, frames and operator theory, in: Contemp. Math. 345, Amer. Math. Soc., Providence, 2004, 87-113.

[6] J. Dixmier, $C^{*}$-algebras, North-Holland Publishing Co., Amsterdam-New YorkOxford, 1977.

[7] M. Frank and D. R. Larson, A module frame concept for Hilbert $C^{*}$-modules, in: Contemp. Math. 247, Amer. Math. Soc., Providence, 1999, 207-233.

[8] M. Frank and D. R. Larson, Frames in Hilbert $C^{*}$-modules and $C^{*}$-algebras, J. Operator Theory 48 (2002), 273-314.

[9] M. Frank, V. I. Paulsen and T. R. Tiballi, Symmetric approximation of frames and bases in Hilbert spaces, Trans. Amer. Math. Soc. 354 (2002), 777-793.

[10] C. Lance, Hilbert $C^{*}$-modules. A Toolkit for Operator Algebraists, Cambridge University Press, Cambridge, 1995.

[11] B. Magajna, Hilbert $C^{*}$-modules in which all closed submodules are complemented, Proc. Amer. Math. Soc. 125 (1997), 849-852.

[12] I. Raeburn and S. J. Thompson, Countably generated Hilbert modules, the Kasparov stabilisation theorem, and frames in Hilbert modules, Proc. Amer. Math. Soc. 131 (2003), 1557-1564. 
[13] J. Schweizer, A description of Hilbert $C^{*}$-modules in which all closed submodules are orthogonally closed, Proc. Amer. Math. Soc. 127 (1999), 2123-2125.

[14] N. E. Wegge-Olsen, $K$-theory and $C^{*}$-algebras. A Friendly Approach, The Clarendon Press, Oxford University Press, New York, 1993.

Lj. Arambašić

Department of Mathematics

University of Zagreb

Bijenička 30, 10000 Zagreb

Croatia

E-mail: ljsekul@math.hr

Received: 10.4.2006. 